\title{
Evidence for Flash Mixing in He-rich sdB Stars
}

\author{
Allen V. Sweigart and Thierry Lanz \\ NASA Goddard Space Flight Center, Code 681, Greenbelt, MD 20771, USA
}

Thomas M. Brown

Space Telescope Science Institute, 3700 San Martin Drive, Baltimore, MD 21218, USA

Ivan Hubeny

National Optical Astronomy Observatory, PO Box 26732, Tucson, AZ 85726, USA

Wayne B. Landsman

NASA Goddard Space Flight Center, Code 681, Greenbelt, MD 207r1, USA

\begin{abstract}
We present FUSE spectra of three He-rich sdB stars. Two of these stars, PG1544+488 and JL87, reveal extremely strong C III lines, suggesting that they have mixed triple- $\alpha$ carbon from the deep interior out to their surfaces. Using TLUSTY NLTE line-blanketed model atmospheres, we find that PG1544+488 has a surface composition of $96 \% \mathrm{He}, 2 \% \mathrm{C}$, and 1\% N. JL87 shows a similar surface enrichment of $\mathrm{C}$ and $\mathrm{N}$ but still retains a significant amount of hydrogen. In contrast, the third star, LB1766, is devoid of hydrogen and strongly depleted of carbon, indicating that its surface material has undergone CN-cycle processing.

We interpret these observations with new evolutionary calculations which suggest that He-rich sdB stars with C-rich compositions arise from a delayed helium-core flash on the white-dwarf cooling curve. During such a flash the interior convection zone will penetrate into the stellar envelope, thereby mixing the envelope with the He- and C-rich core. Such "flash-mixed" stars will arrive on the extreme horizontal branch (EHB) with He- and C-rich surface compositions and will be hotter than the hottest canonical EHB stars. Two types of flash mixing are possible: "deep" and "shallow", depending on whether the hydrogen envelope is mixed deeply into the site of the helium flash or only with the outer layers of the core. Based on both their stellar parameters and surface compositions, we suggest that PG1544+488 and JL87 are examples of "deep" and "shallow" flash mixing, respectively.
\end{abstract}

\section{Introduction}

Extreme horizontal-branch (EHB) stars occupy the hot end of the horizontal branch (HB) and are distinguished by their high effective temperatures, $\mathrm{T}_{\text {eff }}>20,000 \mathrm{~K}$, and high surface gravities, $\log \mathrm{g}>5$. In the Galactic field they correspond to the subdwarf B (sdB) stars, while in the globular clusters they lie at the faint end of the blue HB tail. Most sdB stars are extremely deficient in helium, but a minority are helium-rich. Deficiencies in helium have been attributed to gravitational settling, but the helium-rich members of the class, especially those with enhanced carbon, present a puzzling exception.

(c) 2019 Kluwer Academic Publishers. Printed in the Netherlands. 
Here we explore the origins of the He-rich sdB stars in light of new Far Ultraviolet Spectroscopic Explorer (FUSE) observations of three field He-sdB stars and new theoretical calculations for the helium flash.

\section{FUSE Observations and Spectroscopic Analysis}

We selected three He-rich sdB stars previously studied with optical spectroscopy: PG1544+488, JL87 and LB1766. PG1544+488 is the class prototype and is nearly devoid of hydrogen. JL87 is moderately enriched in helium, $\mathrm{He} / \mathrm{H} \sim 0.1-0.2$ by number. Optical spectra suggest that these two stars have similar effective temperatures $\mathrm{T}_{\text {eff }} \sim$ 30,000 K. LB1766 shows no evidence for hydrogen. Unlike the other two stars, its spectrum does not suggest strong carbon enrichment.

We analyzed our FUSE spectra with NLTE line-blanketed TLUSTY model atmospheres which included 741 individual levels of H I-II, He IIII, C II-V, N II-VI and Si III-V grouped into 301 NLTE superlevels. We used lines of minor ions ( $\mathrm{Si}$ III, C II, C IV) to determine $\mathrm{T}_{\text {eff }}$ and lines of dominant ions (C III, N III, Si IV) to derive the surface abundances. Surface gravities were derived by matching the wings of the Lyman and He II lines. Interstellar line absorption was added to the photospheric spectrum in order to match the interstellar features.

Our results, given in Table 1, confirm the high $\mathrm{C}$ abundances of PG1544+488 and JL87 suggested by previous optical studies. Both

stars show evidence for the dredge-up of triple- $\alpha$ C. The surface of PG1544+488 is virtually devoid of $\mathrm{H}$, while JL87 has a significant amount of $\mathrm{H}$ in its atmosphere. The third star LB1766 is also devoid of $\mathrm{H}$ like PG1544+488, but its atmosphere is strongly depleted in C. The surface composition of LB1766 is consistent with CN-cycle processing.

Table I. Results from analysis of FUSE spectra

\begin{tabular}{clll}
\hline \multicolumn{1}{c}{ Star } & PG1544+488 & \multicolumn{1}{c}{ JL87 } & \multicolumn{1}{c}{ LB1766 } \\
\hline Stellar parameters: & & & \\
$\mathrm{T}_{\text {eff }}[\mathrm{K}]$ & $36000 \pm 2000$ & $29000 \pm 2000$ & $40000 \pm 2000$ \\
$\log \mathrm{g}\left[\mathrm{cm} / \mathrm{s}^{2}\right]$ & $6.0 \pm 0.3$ & $5.5 \pm 0.3$ & $6.3 \pm 0.3$ \\
Surface abundances & (mass fraction): & & \\
$\mathrm{H}$ & $<0.002$ & $0.55-0.70$ & 0.0025 \\
$\mathrm{He}$ & 0.96 & $0.43-0.28$ & 0.99 \\
$\mathrm{C}$ & 0.02 & 0.014 & 0.0001 \\
$\mathrm{~N}$ & 0.01 & 0.004 & 0.006 \\
\hline
\end{tabular}




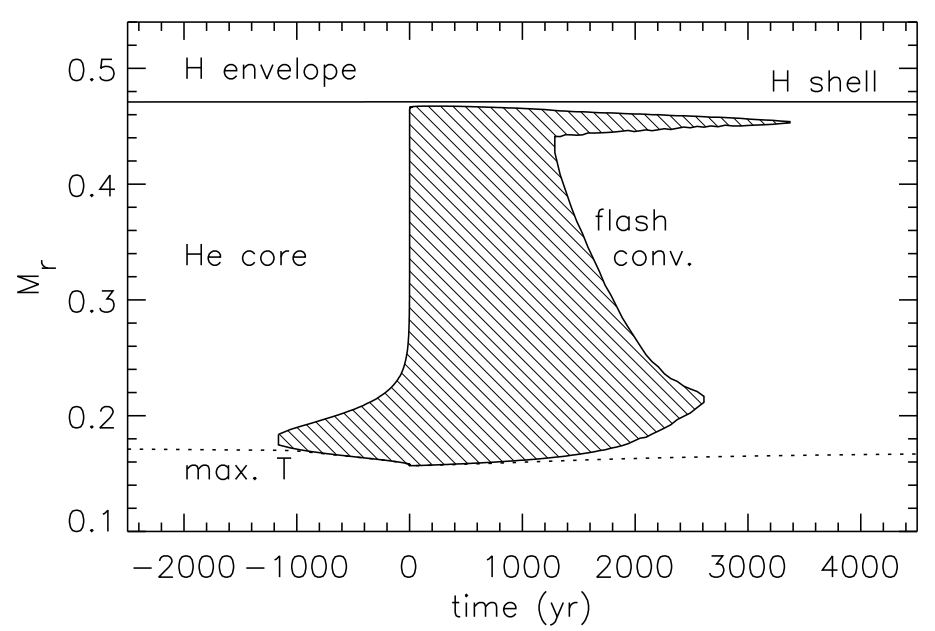

Figure 1. Time dependence of the flash convection zone (shaded area) during a canonical helium flash. The zero-point of the timescale corresponds to the peak of the flash. The ordinate gives the mass coordinate $M_{r}$ in solar units. The helium flash occurs off-center at the point of maximum temperature (dotted curve). During a canonical flash the flash convection does not reach into the hydrogen envelope, and the surface composition of the star is unchanged.

\section{Evolutionary Interpretation: Flash Mixing}

Low-mass stars which undergo very high mass loss on the red-giant branch can evolve to high effective temperatures before igniting helium in their cores, leading to the so-called "hot flashers" (see, e.g., Brown et al., 2001). The high helium-burning luminosity during the helium flash $\left(\gtrsim 10^{9} L_{\odot}\right)$ produces a temporary convection zone that extends from the site of the helium flash outward to just inside the base of the hydrogen envelope (see Figure 1). Following the flash peak, this convection zone retreats and disappears although a small convective shell persists in the outer part of the core for a few thousand years. Normally the flash convection zone does not penetrate into the envelope due to the high entropy barrier of the hydrogen shell. However, such penetration becomes inevitable if the helium flash occurs on the whitedwarf cooling curve, where the hydrogen shell is much weaker. During the ensuing "flash mixing", helium and carbon from the core will be carried outward into the envelope while hydrogen from the envelope will be mixed into the core.

Our calculations have revealed two types of flash mixing, depending on where the flash occurs along the white-dwarf cooling curve (see Lanz et al., 2003 for a fuller discussion): 


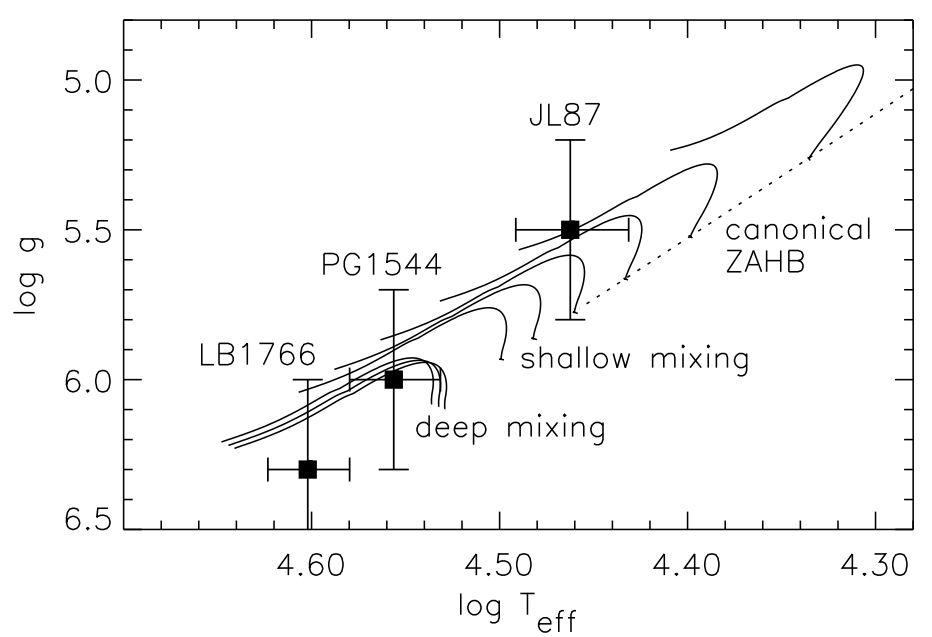

Figure 2. Comparison of the stellar parameters for the He-rich sdB stars PG1544+488, JL87 and LB1766 with EHB evolutionary tracks for canonical models (reddest 4 tracks), models with shallow mixing (intermediate 2 tracks) and models with deep mixing (bluest 3 tracks). The dotted line denotes the canonical ZAHB.

- "Deep" mixing: If flash mixing occurs when the core is fully convective outside the flash site, then the envelope $\mathrm{H}$ will be mixed into the hot He-burning regions and rapidly burned. The resulting EHB star will be enriched in $\mathrm{He}$ and $\mathrm{C}$ and greatly depleted in $\mathrm{H}$.

- "Shallow" mixing: If flash mixing occurs later when there is a distinct convective shell in the outer part of the core, the envelope $\mathrm{H}$ will not be mixed deeply and thus will not be burned. The EHB star will again be He- and C-rich but will also have significant $\mathrm{H}$.

Figure 2 compares the stellar parameters for our three He-rich sdB stars with EHB evolutionary tracks with flash mixing. Within the errors both the stellar parameters and surface compositions of PG1544+488 and JL87 agree with those predicted for stars with deep and shallow flash mixing, respectively. Flash mixing may therefore represent a new evolutionary channel for producing He-rich sdB with enhanced C. However, LB1766 must have had a very different evolutionary history.

\section{References}

Brown, T. M., Sweigart, A. V., Lanz, T., Landsman, W. B., \& Hubeny, I.: 2001, ApJ 562, 368

Lanz, T., Brown, T. M., Sweigart, A. V., Hubeny, I., \& Landsman, W. B.: 2003, ApJ, submitted (astro-ph/0308440) 